\title{
A Study of Students' Attitude towards Peace
}

\author{
Prof. K. S. Misra* \\ Dean, Faculty of Arts, University of Allahabad, Prayagraj, Uttar Pradesh, India \\ *Corresponding Author \\ Prof. K. S. Misra \\ Article History \\ Received: 08.07.2019 \\ Accepted: 18.07.2019 \\ Published: 30.07 .2019
}

\begin{abstract}
Differences between global and local; society and individual; traditional cultural norms and modernity; long-term and short-term goals; pressures for competition and provisions for equality of opportunities; growth of knowledge and our ability to assimilate and accommodate it; and spiritual and materialistic values often lead to conflicts and disturb the peaceful climate in the society as well as inner peace of mind.. Nan-Zhao (2004) stated "A culture of peace reflects 'active, positive, participatory process where diversity is respected, difference is tolerated, dialogue is encouraged, and conflicts solved in a spirit of mutual understanding and co-operation' Culture of peace is needs a transformation of values, attitudes and behaviours. Education can develop favourable attitudes towards peace among students living in a complex society. The present study has been done to study peace-attitudes of students studying in high school and Ph.D. classes.
\end{abstract}

Keywords: Attitude, Peace, Education

\section{INTRODUCTION}

Objectives of the study: Following objectives have been formulated-

- To construct a Peace Attitude Scale.

- To compare peace attitude of students studying in high school and Ph.D. classes.

\section{PROCEDURE}

An attitude scale was constructed based on Likert's method for measuring students' Attitude towards peace. Comparisons were made using t-ratio.

\section{CONSTRUCTION OF PEACE ATTITUDE SCALE}

To begin with the researcher constructed an attitude scale for measuring students' attitude towards peace. Items were prepared on the basis of researcher's experience in conflict resolution strategies. The try-out form consisted of 39 statements. It had 17 negative items while 22 items were positive. The five alternate responses were 'Strongly agree, agree, undecided, disagree, and Strongly disagree'. The try-out form was administered on a sample og 240 students of IX and X classes and 60 students enrolled in different departments of the University of Allahabad. Scoring was done by awarding a score of 5, 4, 3, 2, and 1 for these responses respectively. All scores were added together to get a score for peace attitude. Firstly after scoring scales filled up by 240 students were taken. They were arranged in an ascending order of scores on the Peace Attitude Scale. Then $27 \%$ of the sheets i.e. 65 , were selected from either ends. Thereafter t-ratios were computed. They have been given in table 1. A look at the table shows that ten values of t-ratios are not significant at .05 level. These items were not included in the final form of the Peace Attitude Scale (PAS). Chi-squares were also computed. They have been shown in table 2. Perusal of the table shows that five chi-squares are not significant at .05 level. They were rejected for inclusion in the final form of PAS. In this way 13 items were rejected. Thus, the final form consists of 26 items. Items at s.no.1,2, 3, 5, 8, 11, 13, 16, 17, 19, 22, 23, and 26. Are negative and the remaining 13 items are positive.

Copyright @ 2019: This is an open-access article distributed under the terms of the Creative Commons Attribution license which permits unrestricted use, distribution, and reproduction in any medium for non commercial use (NonCommercial, or CC-BY-NC) provided the original author and source are credited. 
Table-1: Showing means, standard deviations and t-ratios for various items of the Peace Attitude Scale

\begin{tabular}{|c|c|c|c|c|c|}
\hline \multirow[t]{2}{*}{ Item no. in try-out form } & \multicolumn{2}{|l|}{ Means for } & \multicolumn{2}{|c|}{ Standard Deviations for } & \multirow[t]{2}{*}{ t-ratio } \\
\hline & High group & Low group & High group & Low group & \\
\hline 1 & 3.7385 & 2.4154 & 1.1494 & 1.4884 & $5.6722^{*}$ \\
\hline 2 & 3.0923 & 2.1385 & 1.5178 & 1.3906 & $3.7358^{*}$ \\
\hline 3 & 3.6154 & 2.5692 & 1.2953 & 1.4574 & $4.3256^{*}$ \\
\hline 4 & 4.1538 & 3.4308 & 0.9879 & 1.3106 & $3.5519^{*}$ \\
\hline 5 & 2.7538 & 2.0308 & 1.4581 & 1.2621 & $3.0230^{*}$ \\
\hline 6 & 3.6154 & 3.0154 & 1.4544 & 1.453 & $2.3449^{*}$ \\
\hline 7 & 4.6615 & 4.000 & 0.6441 & 1.1859 & $3.9522^{*}$ \\
\hline 8 & 3.5692 & 2.9077 & 1.4358 & 1.3077 & $2.7463^{*}$ \\
\hline 9 & 2.8462 & 2.5385 & 1.1889 & 1.2879 & 1.1453 \\
\hline 10 & 2.7385 & 2.2154 & 1.3143 & 1.3404 & $2.2465^{*}$ \\
\hline 11 & 2.5538 & 2.7077 & 1.5414 & 1.5075 & 0.5753 \\
\hline 12 & 3.4462 & 2.7385 & 1.4687 & 1.5740 & $2.6203^{*}$ \\
\hline 13 & 3.3077 & 2.8000 & 1.4244 & 1.4383 & $2.0220^{*}$ \\
\hline 14 & 3.4923 & 4.2769 & 1.0534 & 1.5524 & $3.3718^{*}$ \\
\hline 15 & 3.9692 & 2.3538 & 1.1854 & 1.3161 & $7.3526^{*}$ \\
\hline 16 & 4.0615 & 3.4000 & 0.8817 & 1.2349 & $3.5150^{*}$ \\
\hline 17 & 2.8615 & 2.7231 & 1.3906 & 1.4844 & 0.5488 \\
\hline 18 & 4.3692 & 2.4308 & 1.0242 & 1.4681 & $8.7306^{*}$ \\
\hline 19 & 3.7692 & 3.4923 & 1.2218 & 1.4375 & 1.1834 \\
\hline 20 & 3.5385 & 3.2462 & 1.1467 & 1.5003 & 1.2480 \\
\hline 21 & 2.2615 & 2.1538 & 1.3379 & 1.2403 & 0.4759 \\
\hline 22 & 4.2462 & 3.7538 & 0.7912 & 1.0757 & $2.9723^{*}$ \\
\hline 23 & 4.4000 & 3.7538 & 0.9152 & 1.2873 & $3.2982^{*}$ \\
\hline 24 & 2.9385 & 2.9077 & 1.4456 & 1.4331 & 0.1219 \\
\hline 25 & 3.3462 & 2.8923 & 1.3742 & 1.5013 & $2.9862^{*}$ \\
\hline 26 & 4.6769 & 3.5231 & 0.9033 & 1.4589 & $5.4215^{*}$ \\
\hline 27 & 4.0308 & 3.1846 & 1.2621 & 1.3907 & $3.6325^{*}$ \\
\hline 28 & 3.8154 & 2.8154 & 1.1577 & 1.2234 & $4.7866^{*}$ \\
\hline 29 & 3.9538 & 3.2923 & 0.9088 & 1.2957 & $3.3700^{*}$ \\
\hline 30 & 4.0923 & 2.8769 & 1.2083 & 1.5763 & $4.9336^{*}$ \\
\hline 31 & 4.2000 & 2.5846 & 0.9552 & 1.5400 & $7.1866^{*}$ \\
\hline 32 & 3.2462 & 2.6308 & 1.3697 & 1.4314 & $2.5043^{*}$ \\
\hline 33 & 4.2923 & 3.8000 & 0.9138 & 1.1347 & $2.7243^{*}$ \\
\hline 34 & 3.6923 & 2.3231 & 1.3101 & 1.3475 & $5.8737^{*}$ \\
\hline 35 & 3.6923 & 3.4615 & 1.0595 & 1.3000 & 1.1094 \\
\hline 36 & 3.2000 & 2.9846 & 1.3370 & 1.4196 & 0.8905 \\
\hline 37 & 2.6000 & 2.9231 & 1.3323 & 1.5442 & 1.2771 \\
\hline 38 & 3.6769 & 2.8308 & 1.1195 & 1.4850 & $3.6682^{*}$ \\
\hline 39 & 4.3231 & 3.000 & 1.1334 & 1.4252 & $5.8579^{*}$ \\
\hline
\end{tabular}

${ }^{*}$ Significant at .05 level 
K. S. Misra.; South Asian Res J Human Soc Sci; Vol-1, Iss- 1 (Jun-Jul, 2019): 5-9

Table-2: Values of frequencies for various responses and chi-square for various items of the Peace Attitude Scale for the sample of secondary level students

\begin{tabular}{|c|c|c|c|c|c|c|c|}
\hline \multirow{2}{*}{$\begin{array}{l}\text { Item no. in } \\
\text { try-out form }\end{array}$} & \multicolumn{5}{|c|}{ Frequencies for responses } & \multirow[t]{2}{*}{ Chi-square } & \multirow{2}{*}{$\begin{array}{l}\text { Item no. in } \\
\text { final form }\end{array}$} \\
\hline & $\begin{array}{l}\text { Strongly } \\
\text { disagree }\end{array}$ & Disagree & undecided & Agree & $\begin{array}{l}\text { Strongly } \\
\text { agree }\end{array}$ & & \\
\hline 1 & 46 & 48 & 26 & 73 & 47 & $23.208^{* *}$ & 1 \\
\hline 2 & 77 & 66 & 21 & 47 & 29 & $47.000^{* *}$ & 2 \\
\hline 3 & 42 & 65 & 21 & 63 & 49 & $26.667^{* *}$ & 3 \\
\hline 4 & 11 & 37 & 30 & 85 & 77 & $83.833^{* *}$ & 4 \\
\hline 5 & 80 & 63 & 33 & 43 & 21 & $46.417^{* *}$ & 5 \\
\hline 6 & 37 & 38 & 40 & 62 & 63 & $14.708^{* *}$ & 6 \\
\hline 7 & 7 & 7 & 21 & 64 & 141 & $270.750^{* *}$ & 7 \\
\hline 8 & 42 & 54 & 56 & 47 & 51 & 1.792 & \\
\hline 9 & 48 & 66 & 65 & 39 & 22 & $28.542^{* *}$ & \\
\hline 10 & 69 & 65 & 33 & 45 & 18 & $48.000^{* *}$ & 8 \\
\hline 11 & 80 & 42 & 34 & 45 & 39 & $28.042^{* *}$ & \\
\hline 12 & 57 & 37 & 34 & 56 & 56 & $10.958^{*}$ & 9 \\
\hline 13 & 48 & 57 & 47 & 43 & 45 & 2.417 & \\
\hline 14 & 20 & 20 & 19 & 70 & 111 & $142.958^{* *}$ & 10 \\
\hline 15 & 41 & 38 & 23 & 80 & 58 & $39.542^{* *}$ & 11 \\
\hline 16 & 10 & 13 & 76 & 77 & 64 & $94.792^{* *}$ & 12 \\
\hline 17 & 43 & 72 & 32 & 51 & 42 & $18.792^{\star *}$ & \\
\hline 18 & 37 & 26 & 27 & 58 & 92 & $64.208^{* *}$ & 13 \\
\hline 19 & 23 & 31 & 29 & 91 & 66 & $71.833^{* *}$ & \\
\hline 20 & 22 & 47 & 39 & 83 & 49 & $41.333^{* *}$ & \\
\hline 21 & 86 & 70 & 35 & 34 & 15 & $70.458^{* *}$ & \\
\hline 22 & 2 & 19 & 41 & 99 & 79 & $136.833^{* *}$ & 14 \\
\hline 23 & 12 & 11 & 19 & 82 & 116 & $193.458^{* *}$ & 15 \\
\hline 24 & 49 & 51 & 47 & 47 & 46 & 0.333 & \\
\hline 25 & 31 & 47 & 27 & 63 & 72 & $31.917^{* *}$ & 16 \\
\hline 26 & 19 & 24 & 12 & 46 & 139 & $229.125^{\star *}$ & 17 \\
\hline 27 & 30 & 26 & 28 & 74 & 72 & $44.167^{* *}$ & 18 \\
\hline 28 & 23 & 53 & 71 & 54 & 39 & $27.000^{* *}$ & 19 \\
\hline 29 & 13 & 25 & 52 & 97 & 53 & $87.417^{\star *}$ & 20 \\
\hline 30 & 34 & 44 & 23 & 54 & 85 & $46.708^{\star *}$ & 21 \\
\hline 31 & 36 & 28 & 24 & 72 & 70 & $39.167^{* *}$ & 22 \\
\hline 32 & 41 & 76 & 32 & 51 & 40 & $24.208^{* *}$ & 23 \\
\hline 33 & 7 & 14 & 25 & 100 & 94 & $170.542^{* *}$ & 24 \\
\hline 34 & 49 & 50 & 46 & 55 & 40 & 2.542 & \\
\hline 35 & 14 & 33 & 52 & 58 & 53 & $62.958^{* *}$ & \\
\hline 36 & 31 & 53 & 43 & 68 & 45 & $15.583^{* *}$ & \\
\hline 37 & 46 & 60 & 36 & 55 & 43 & 7.625 & \\
\hline 38 & 28 & 44 & 58 & 69 & 41 & $20.958^{* *}$ & 25 \\
\hline 39 & 20 & 29 & 36 & 64 & 91 & $70.708^{* *}$ & 26 \\
\hline
\end{tabular}

*Significant at .05 level 
Table-3: Values of frequencies for various responses and chi-square for various items of the Peace Attitude Scale for the sample of Ph.D. students

\begin{tabular}{|c|c|c|c|c|c|c|}
\hline \multirow{2}{*}{ Item no. in try-out form } & \multicolumn{5}{|c|}{ Frequencies for responses } & \multirow[b]{2}{*}{ Chi-square } \\
\hline & $\begin{array}{l}\text { Strongly } \\
\text { disagree }\end{array}$ & Disagree & undecided & Agree & Strongly agree & \\
\hline 1 & 3 & 11 & 7 & 21 & 19 & 19.41 \\
\hline 2 & 12 & 22 & 5 & 18 & 4 & 20.393 \\
\hline 3 & 0 & 3 & 8 & 30 & 20 & 29.033 \\
\hline 4 & 5 & 6 & 7 & 32 & 11 & 41.869 \\
\hline 5 & 13 & 32 & 4 & 8 & 4 & 44.656 \\
\hline 6 & 3 & 3 & 10 & 36 & 9 & 61.541 \\
\hline 7 & 8 & 3 & 2 & 32 & 16 & 50.23 \\
\hline 8 & 4 & 4 & 4 & 35 & 14 & 59.41 \\
\hline 9 & 6 & 26 & 5 & 18 & 6 & 28.918 \\
\hline 10 & 4 & 10 & 11 & 21 & 15 & 13.016 \\
\hline 11 & 15 & 23 & 7 & 10 & 6 & 15.967 \\
\hline 12 & 7 & 23 & 15 & 11 & 5 & 16.787 \\
\hline 13 & 8 & 31 & 8 & 11 & 3 & 38.918 \\
\hline 14 & 5 & 1 & 6 & 42 & 7 & 92.689 \\
\hline 15 & 10 & 37 & 6 & 5 & 3 & 65.148 \\
\hline 16 & 5 & 3 & 8 & 36 & 9 & 59.902 \\
\hline 17 & 3 & 15 & 10 & 26 & 7 & 25.803 \\
\hline 18 & 1 & 7 & 8 & 30 & 15 & 40.557 \\
\hline 19 & 14 & 13 & 10 & 23 & 1 & 20.557 \\
\hline 20 & 2 & 9 & 11 & 34 & 5 & 52.689 \\
\hline 21 & 3 & 17 & 4 & 26 & 11 & 30.066 \\
\hline 22 & 3 & 2 & 6 & 40 & 10 & 82.361 \\
\hline 23 & 5 & 2 & 6 & 34 & 14 & 55.148 \\
\hline 24 & 16 & 23 & 7 & 12 & 3 & 19.902 \\
\hline 25 & 7 & 23 & 11 & 13 & 7 & 14.164 \\
\hline 26 & 2 & 5 & 5 & 22 & 27 & 42.852 \\
\hline 27 & 6 & 4 & 5 & 25 & 21 & 32.689 \\
\hline 28 & 4 & 12 & 10 & 25 & 10 & 19.738 \\
\hline 29 & 3 & 4 & 8 & 34 & 12 & 52.852 \\
\hline 30 & 9 & 11 & 3 & 25 & 13 & 21.377 \\
\hline 31 & 14 & 31 & 7 & 6 & 3 & 41.541 \\
\hline 32 & 10 & 13 & 5 & 25 & 8 & 19.574 \\
\hline 33 & 3 & 4 & 7 & 39 & 8 & 74.984 \\
\hline 34 & 14 & 27 & 9 & 5 & 6 & 26.459 \\
\hline 35 & 2 & 8 & 17 & 29 & 5 & 39.246 \\
\hline 36 & 6 & 14 & 10 & 22 & 9 & 12.525 \\
\hline 37 & 4 & 21 & 12 & 19 & 5 & 19.902 \\
\hline 38 & 4 & 1 & 14 & 37 & 5 & 70.721 \\
\hline 39 & 4 & 4 & 5 & 20 & 28 & 143.042 \\
\hline
\end{tabular}

The same PAS was administered on students enrolled in Ph.D. class. Chi-squares were calculated. They have been presented in table 3 . The perusal of the table shows that all vaslues are significant at .05 level. So, all items were retained in the final form.

\section{Reliability and validity}

Alpha and split half reliability for the PAS are .718 and .807 for the sample of research students. For the students of ninth class they are .623 and .703 . 
Table-4: Item-total correlations for Peace Attitude Scale for students of class IX and Ph,D.

\begin{tabular}{|r|c|c|r|r|c|}
\hline \multirow{2}{*}{ Item no. } & \multicolumn{2}{|c|}{ Item-total correlations for } & \multicolumn{2}{|c|}{ Item no. } & \multicolumn{2}{|c|}{ Item-total correlations for } \\
\cline { 2 - 3 } & $\begin{array}{c}\text { IX class } \\
(\mathrm{N}=80)\end{array}$ & $\begin{array}{c}\text { Ph.D. class } \\
(\mathrm{N}=40)\end{array}$ & & & Ph.D. class \\
\hline 1 & .309 & .358 & 14 & .289 & 580 \\
\hline 2 & .281 & .389 & 15 & .246 & 750 \\
\hline 3 & .311 & .406 & 16 & .299 & 386 \\
\hline 4 & .232 & .655 & 17 & .372 & 365 \\
\hline 5 & .294 & .361 & 18 & .239 & 748 \\
\hline 6 & .249 & .739 & 19 & .305 & 339 \\
\hline 7 & .258 & .724 & 20 & .257 & 604 \\
\hline 8 & .268 & .351 & 21 & .364 & 620 \\
\hline 9 & .267 & .392 & 22 & .438 & .349 \\
\hline 10 & .267 & .620 & 23 & .260 & .352 \\
\hline 11 & .476 & .345 & 24 & .239 & 513 \\
\hline 12 & .286 & .521 & 25 & .284 & .740 \\
\hline 13 & .492 & .634 & 26 & .421 & .536 \\
\hline
\end{tabular}

Item validity was ascertained by finding out correlation between score on the PAS and each item of it for the sample of ninth and Ph. D. students. The values of item-total correlations have been given in table 4 . For ninth class students they ranged from .232 to .421 for IX class students and .349 to .750 for research students. Face validity was ascertained by experts' opinion.

\section{Study of differences in peace attitudes of students of ix and ph.d classes}

Table-5: Means, Standard Deviations, and t-ratio showing differences between students of class IX and Ph,D.

\begin{tabular}{|l|l|l|l|l|l|}
\hline GRADE & N & Mean & Std. Deviation & Std. Error Mean & t-ratio \\
\hline Ph. D. & 40 & 88.8000 & 10.72309 & 1.69547 & \multirow{2}{*}{1.714} \\
\hline 9 & 80 & 92.2750 & 10.33977 & 1.15602 & \\
\hline
\end{tabular}

It was hypothesized that students of ninth and Ph.D. classes do not significantly differ on their peace attitudes. Mean and standard deviation for students of ninth class are 92.275 and 10.33977 respectively. Mean and standard deviation for students of ninth class are 88.80 and 1.72309 respectively. The value of t-ratio is 1.714 which is not significant at .05 level. So, the null hypothesis can be accepted and it can be inferred that students of Ph.D. do not differ from students of Ph.D. on their peace attitudes. When means are divided by no. of items in the tool, it is found that students of both the classes have favourable attitudes toward peace. It means that with the passage of time or as a result of higher education and research, there occurs no change in the peace attitude of students of both the groups. Students understand the need of peace and the process of socialization in the Indian set-up along with pressures of sanskritization and modernization, students' attitude remain equally favourable. However, it implies the need of peace education at Ph. D. level so as to make students' attitudes towards peace more favourable.

\section{REFERENCES}

1. "UNESCO and a Culture of Peace: Promoting a Global Movement" Retrieved from http://www.unesco.org/general/eng/publish/order.html

2. Education is foundation for lasting peace, UNESCO. Retrieved from www.newsforafrica.com/.../999395190307999395-educationis-foundati. 